\title{
Polarization behaviors in a transverse Ising film with multi-surface layers
}

\author{
QiuShi Li ${ }^{1, a *}$ \\ ${ }^{1}$ College of Mechanical Engineering, Linyi University, Linyi 276005, China \\ aliqiushi@lyu.edu.cn
}

Keywords: ferroelectrics, transverse Ising film, Curie temperature, polarization

\begin{abstract}
The mean-field theory is used to study polarization behaviors in a ferroelectric thin film described by the transverse Ising model. The dependence of average polarization and layer polarizations of the transverse Ising film with two surface layers on the Curie temperature or transverse field is discussed in detail. The numerical calculations are also compared with those of the effective-field theory with correlations.
\end{abstract}

\section{Introduction}

Ferroelectric thin films have been receiving considerable attention in experiment and theory for their potential applications in masses of scientific research fields [1]. Theoretically, the transverse Ising model is considered as an excellent candidate to study phase transitions in the ferroelectric thin film with multi-surface layers [2-10]. Within the framework of the mean-field theory, Wang et al. $[2,3]$ and Sy [4] investigated the dependence of Curie temperature on the surface layer number and total layer number as well as interaction parameters. Qu et al. [5] studied the average polarization as the function of temperature for a thin film with two surface layers. Wang et al. [6] discussed the multi-surface modification on the Curie temperature. Yang et al. [7] calculated the phase diagrams and the crossover features of the interaction parameters from the ferroelectric-dominant phase diagram to the paraelectric-dominant phase diagram for a ferroelectric thin film with two surface layers. Soon afterwards, within the framework of the effective-field theory with correlations, Arhchoui et al. [8] studied the phase diagrams of the ferroelectric thin film with two surface layers and paid a special attention to the effect of surface transverse field on phase transitions. Furthermore, Lu et al. [9] derived a general equation for the diagram of an N-layer ferroelectric thin film with two surface layers and analyzed the dependence of the phase diagrams on the exchange interactions and transverse fields as well as the number of layers $\mathrm{N}$. At the same time, the crossover features for various interaction parameters of the N-layer ferroelectric thin film are also obtained. In most of above mention, however, only the rare works have been carried out on the polarization behaviors of a transverse Ising film.

In the present paper, the mean-field theory [2-7] is used to investigate the polarization behaviors in the transverse Ising film with two surface layers. The average polarization and layer polarizations of the thin film are calculated numerically as the functions of the Curie temperature and transverse field. Meanwhile, the comparison with the corresponding results of the effective-field theory with correlations is also made.

\section{Model and formulas}

The transverse Ising film with two surface layers is supposed to be consisted of a simple cubic lattice structure. The schematic diagram is shown in Fig. 1 . The numbers $1,2,3, \ldots, \mathrm{N}$ labeled on the right side of Fig. 1 are the layer indices. The Hamiltonian of the transverse Ising system is [2-10]

$$
H=-\sum_{i} \Omega_{i} S_{i}^{x}-\frac{1}{2} \sum_{\langle i, j\rangle} J_{i j} S_{i}^{z} S_{j}^{z},
$$

where $\mathrm{S}_{\mathrm{i}}{ }^{\mathrm{x}}$ ( or $\mathrm{S}_{\mathrm{i}}{ }^{\mathrm{z}}$ ) is the $\mathrm{x}$ (or $\mathrm{z}$ ) component of the spin-1/2 at site $\mathrm{i}, \Omega_{\mathrm{i}}$ is the transverse field in the $\mathrm{i}$-th layer. $\Omega_{\mathrm{i}}=\Omega_{\mathrm{s}}$ (or $\Omega_{\mathrm{i}}=\Omega_{\mathrm{b}}$ ) when the i-th layer is the surface (or bulk) layer. $\mathrm{J}_{\mathrm{ij}}=\mathrm{J}_{\mathrm{s}}\left(\right.$ or $\mathrm{J}_{\mathrm{ij}}=\mathrm{J}_{\mathrm{b}}$ ) when the 
spins are on the surface (or bulk) layers. In the following, only the nearest-neighbor two-spin interaction is considered.

Under the mean-field theory, the polarization value of pseudo-spin in the i-th layer [2-7] is

$$
\left\langle S_{i}^{z}\right\rangle=\left(H_{i}^{z} / 2\left|H_{i}\right|\right) \tanh \left(\left|H_{i}\right| / 2 k_{B} T\right) \text {, }
$$

where

$$
\begin{aligned}
& H_{i}^{z}=J_{i, i-1}\left\langle S_{i-1}^{z}\right\rangle+4 J_{i i}\left\langle S_{i}^{z}\right\rangle+J_{i, i+1}\left\langle S_{i+1}^{z}\right\rangle, \\
& \left|H_{i}\right|=\sqrt{\left(\Omega_{i}\right)^{2}+\left(H_{i}^{z}\right)^{2}} .
\end{aligned}
$$

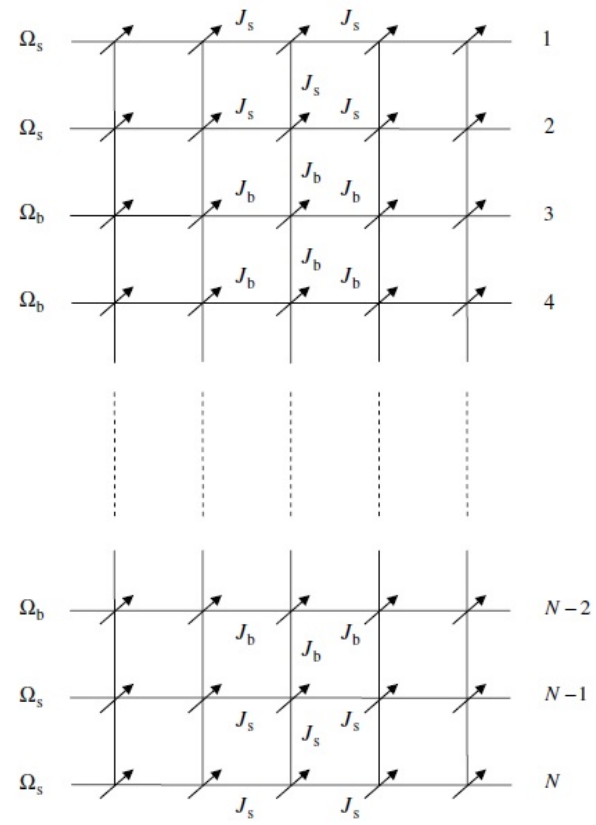

Fig. 1 Schematic diagram of a transverse Ising film with two surface layers.

\section{Numerical results}

The average polarization and layer polarizations of the transverse Ising film as the functions of the Curie temperature or transverse field can be calculated by solving Eqs. 2-4. Similar to Ref. [10], the value of $\Omega_{\mathrm{s}}=\Omega_{\mathrm{b}}=\Omega$ is also adopted for simplicity. Due to the layer symmetry of the transverse Ising film, only N/2 layer polarizations as functions of Curie temperature or transverse field are exhibited in the following figures. All the parameters are assumed to be reduced by $\mathrm{J}$.
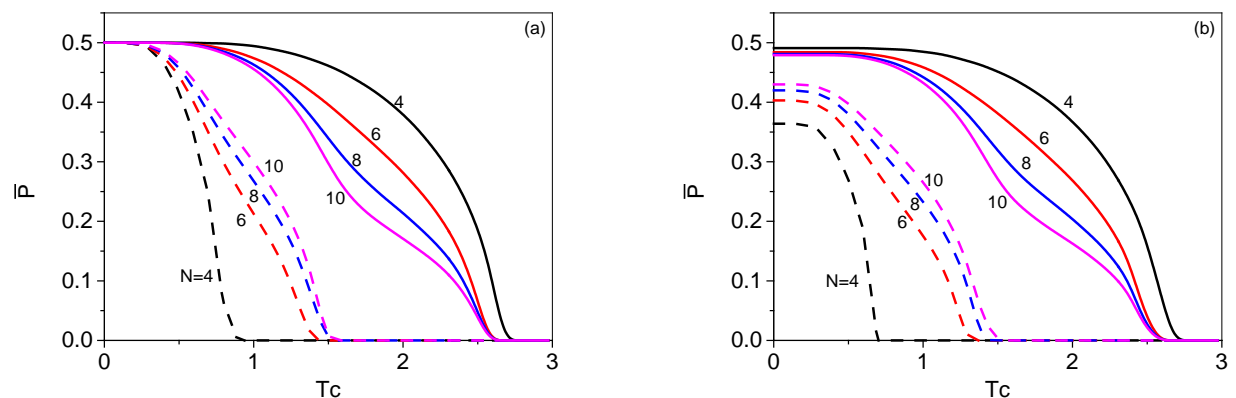

Fig. 2 Average polarization vs. the Curie temperature for (a) $\Omega=0.0$ and (b) $\Omega=1.0$; dash lines: $\mathrm{J}_{\mathrm{s}}=0.5$, solid lines: $\mathrm{J}_{\mathrm{s}}=2.0 ; \mathrm{N}$ denotes the layer number of the transverse Ising film.

Fig. 2 shows the average polarization $\overline{\mathrm{P}}$ vs. the Curie temperature Tc for two transverse fields $\Omega=0.0$ and $\Omega=1.0$ when the layer numbers are selected as $N=4,6,8,10$. It can be found that the 
average polarization $\overline{\mathrm{P}}$ decreases monotonously as the Curie temperature Tc increases. For $\Omega=0.0$ and Tc $=0.0$ in Fig. 2(a), the average polarizations $\overline{\mathrm{P}}$ for the transverse Ising films with different layers of $N=4,6,8$ and 10 are 0.5 (i.e., the saturation polarization $\mathrm{P}_{0}$ ). However, for $\Omega=1.0$ and $\mathrm{Tc}=0.0$ in Fig. 2(b), the average polarizations $\overline{\mathrm{P}}$ are less than 0.5. Meanwhile, the dependence of the average polarization $\overline{\mathrm{P}}$ on the surface exchange interaction $\mathrm{J}_{\mathrm{s}}$ is also shown in Fig. 2. For a certain layer $\mathrm{N}$, the larger the surface exchange interaction $\mathrm{J}_{\mathrm{s}}$, the larger the average polarizations $\overline{\mathrm{P}}$.
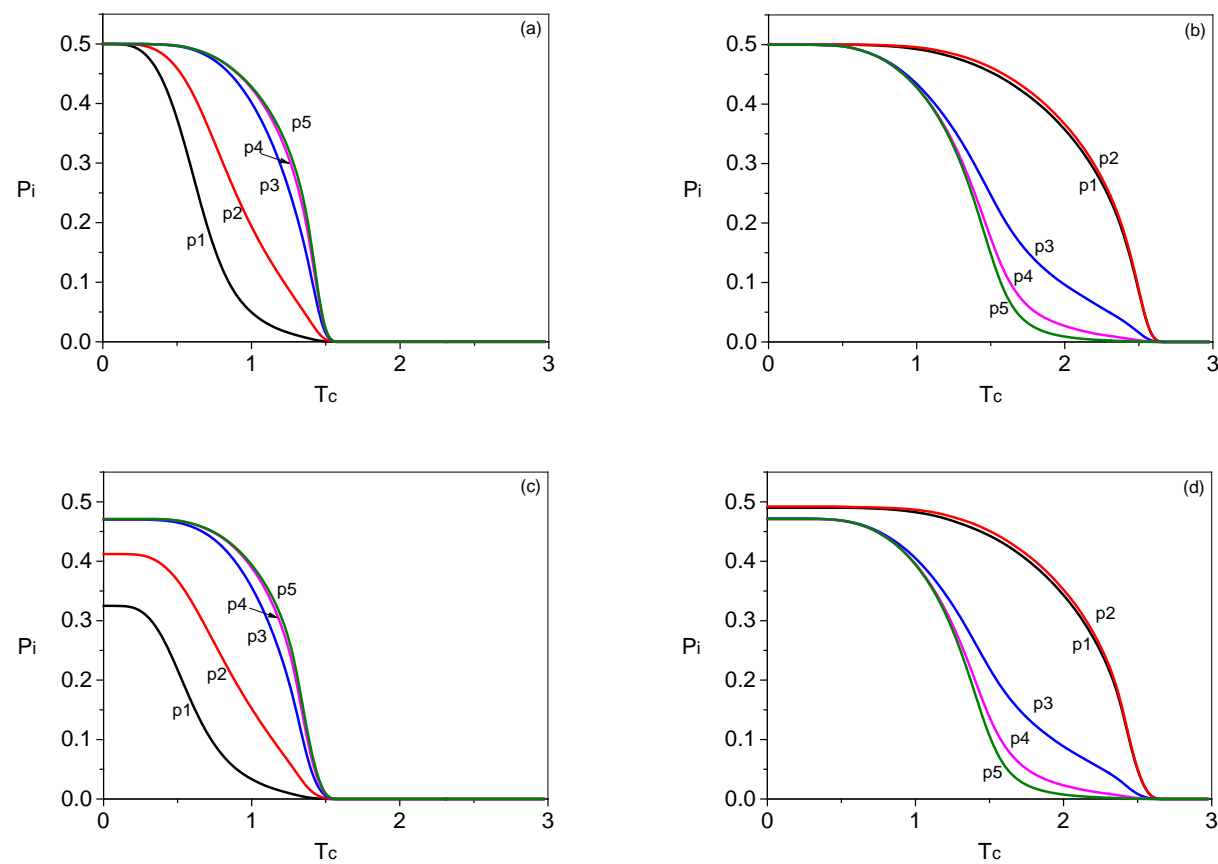

Fig. 3 The layer polarizations vs. the Curie temperature for $N=10$. (a) $\Omega=0.0, \mathrm{~J}_{\mathrm{s}}=0.5$; (b) $\Omega=0.0$, $\mathrm{J}_{\mathrm{s}}=2.0$; (c) $\Omega=1.0, \mathrm{~J}_{\mathrm{s}}=0.5$; (d) $\Omega=1.0, \mathrm{~J}_{\mathrm{s}}=2.0 . \mathrm{P}_{\mathrm{i}}$ denotes the polarization of the i-th layer, and $\mathrm{i}$ is the layer index. Due to the layer symmetry of the transverse Ising film, $\mathrm{i}=1,2,3,4$ and 5.
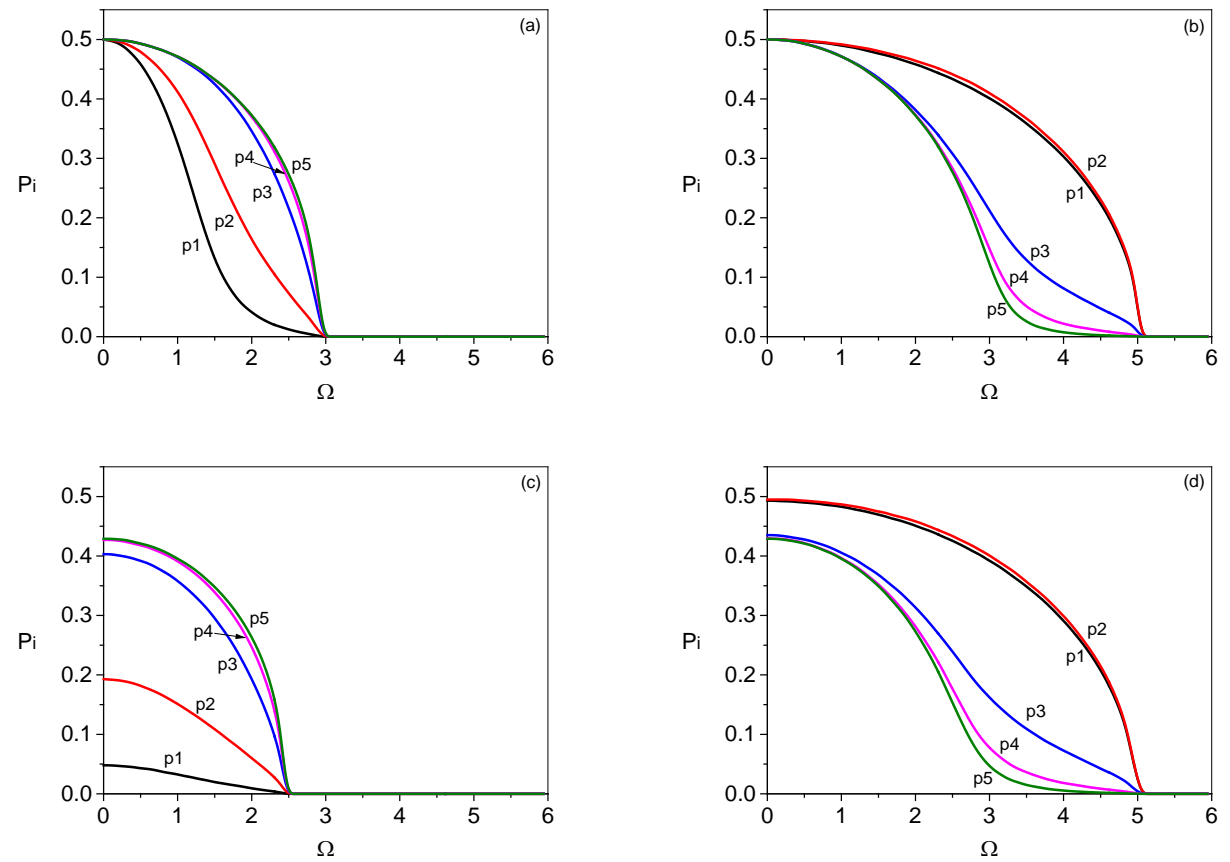

Fig. 4 The layer polarizations vs. the transverse field for $\mathrm{N}=10$. (a) $\mathrm{Tc}=0.0, \mathrm{~J}_{\mathrm{s}}=0.5$; (b) $\mathrm{Tc}=0.0$, $\mathrm{J}_{\mathrm{s}}=2.0$; (c) $\mathrm{Tc}=1.0, \mathrm{~J}_{\mathrm{s}}=0.5$; (d) Tc=1.0, $\mathrm{J}_{\mathrm{s}}=2.0$. $\mathrm{i}$ is the layer index.

Fig. 3 shows the layer polarizations $P_{i}$ vs. the Curie temperature Tc for two transverse fields $\Omega=0.0$ and $\Omega=1.0$ when the layer number is selected as $N=10$. It indicates that the layer polarizations depend 
sensitively on the Curie temperature. With the increase of Curie temperature, the layer polarizations also decrease monotonously. The layer polarizations reduce to zero at the same time. From Figs. 3(a) and 3(b), for $\Omega=0.0$ and $T c=0.0$, it can also be found that the layer polarizations $P_{i}(i=1-5)$ are 0.5 . Meanwhile, for a different $\Omega=1.0$ in Figs. 3(c) and 3(d) are different from Figs. 3(a) and 3(b). The distinctions among these layer polarizations are obvious.

Fig. 4 shows the layer polarizations $P_{i}$ vs. the transverse field $\Omega$ for two Curie temperature Tc $=0.0$ and $\mathrm{Tc}=1.0$ when the layer number is also selected as $\mathrm{N}=10$. It is obvious that the features are similar to the layer polarizations $P_{i}$ vs. the Curie temperature Tc in Fig. 3. These features are similar to the corresponding results of the effective-field theory with correlations in Ref. [10].

\section{Summary}

In this work, the polarization behaviors of the transverse Ising film with two surface layers are studied in detail by the mean-field theory. The dependence of the average polarization and layer polarizations on the Curie temperature or the transverse field is calculated numerically, respectively. Meanwhile, our results are also made a simple comparison with the effective-field theory with correlations.

\section{References}

[1] J.F. Scott, Applications of Modern Ferroelectrics, Science 315 (2007) 954-959.

[2] C.L. Wang, W.L. Zhong, P.L. Zhang, The Curie temperature of ultra-thin ferroelectric films, J. Phys.: Condens. Matter 3 (1992) 4743-4749.

[3] C.L. Wang, S.R.P. Smith, D.R. Tilley, Ferroelectric thin films described by an Ising model in a transverse field, J. Phys.: Condens. Matter 6 (1994) 9633-9646.

[4] H.K. Sy, Surface modification in ferroelectric transitions, J. Phys.: Condens. Matter 5 (1993) 1213-1220.

[5] B.D. Qu, W.L. Zhong, P.L. Zhang, Phase-transition behavior of the spontaneous polarization and susceptibility of ferroelectric thin films, Phys. Rev. B 52 (1995) 766-770.

[6] X.G. Wang, S.H. Pan, G.Z. Yang, Effects of multi-surface modification on Curie temperature of ferroelectric films, J. Phys.: Condens. Matter 11 (1999) 6581-6588.

[7] X. Yang, X.Y. Kuang, C. Lu, Phase transition properties of ferroelectric thin films with two surface layers, Solid State Commun. 139(2006) 397-402.

[8] H. Arhchoui, Y.El. Amraoui, D. Mezzane, I. Luk’yanchuk, Phase diagrams of ferroelectric thin film with two surface layers, Eur. Phys. J. Appl. Phys. 48 (2009) 10503.

[9] Z.X. Lu, B.H. Teng, Y.H. Rong, X.H. Lu, X. Yang, A general analytical equation for phase diagrams of an N-layer ferroelectric thin film with two surface layers, Phys. Scr. 81 (2010) 035004.

[10] X.Z. Wang, X.Y. Jiao, J.J. Wang, Magnetic properties of a transverse Ising film with s=1/2, J. Phys.: Condens. Matter 4 (1992) 3651-3658. 